\title{
Pilot safety and feasibility study of treadmill aerobic exercise in Parkinson disease with gait impairment
}

\author{
Frank M. Skidmore, MD; ${ }^{1-2}$ Shawnna L. Patterson, MD, PhD ${ }^{1}$ Lisa M. Shulman, $M D ;^{2}$ John D. Sorkin, MD, \\ PhD; ${ }^{3}$ Richard F. Macko, MD $^{1-3^{*}}$ \\ ${ }^{1}$ Department of Neurology, Baltimore Department of Veterans Affairs Medical Center (VAMC), and Exercise and \\ Robotics Rehabilitation Research and Development Center, Baltimore, MD; ${ }^{2}$ Department of Neurology, University of \\ Maryland School of Medicine, Baltimore, MD; ${ }^{3}$ Department of Medicine, Division of Gerontology, University of \\ Maryland School of Medicine, and Geriatrics Research, Education, and Clinical Center, Baltimore VAMC, Baltimore, MD
}

\begin{abstract}
This pilot study evaluated the safety and feasibility of a 3-month progressive treadmill aerobic exercise (TM-AEX) program for persons with Parkinson disease with gait impairment. Eight subjects underwent a treadmill stress test to determine eligibility. Of these subjects, three were referred for further cardiac evaluation and five were enrolled. In 136 TM-AEX sessions, 11 falls or near falls and 9 episodes (8 asymptomatic) of systolic blood pressure drops $>20 \mathrm{mmHg}$ occurred. Harness supports prevented injury from falls. TM-AEX significantly improved the subjects' total Unified Parkinson's Disease Rating Scale scores and peak ambulatory workload capacities. This study suggests that an aerobic exercise program is feasible for persons who have Parkinson disease with gait impairment; however, precautions must be taken to prevent falls. Systolic blood pressure instability during exercise points to the need for autonomic dysfunction monitoring. Our data indicate that TM-AEX may reduce symptom severity and improve fitness. Further studies are needed for a better understanding of the risks and benefits of TM-AEX in this population.
\end{abstract}

Key words: aerobic exercise, autonomic instability, falls, fitness, gait dysfunction, Parkinson disease, peak ambulatory work capacity, postural instability, rehabilitation, safety, treadmill exercise, $\mathrm{VO}_{2}$ peak.

\section{INTRODUCTION}

Despite effective symptomatic therapies for Parkinson disease (PD), function declines as patients develop motor fluctuations, gait impairment, and postural instability. Gait abnormalities may persist even when patients are adequately treated [1]. Studies suggest that treadmill walking reduces impairment and disability in PD [2-4]. For example, treadmill walking immediately alters gait patterning in PD, improving gait rhythm and stability more than overground walking [2]. Overground walking speed and stride length improve after as little as 1 month of training [3]. Recently, Protas et al. showed that a gait and step training program decreased frequency of falls and improved gait speed and stride length [4]. However, these prior studies examined numerous complex intervention strategies involving, for example, direction walking and sudden stops and starts during treadmill training (Protas et al. [4]) or training in the context of a complex

Abbreviations: GXT = graded treadmill exercise testing, $\mathrm{HR}=$ heart rate, $\mathrm{HY}=$ Hoehn and Yahr (stage), $\mathrm{MET}=$ metabolic equivalent, $\mathrm{PD}=$ Parkinson disease, $\mathrm{SBP}=$ systolic blood pressure, $\mathrm{TM}-\mathrm{AEX}=$ treadmill aerobic exercise, $\mathrm{UPDRS}=$ Unified Parkinson's Disease Rating Scale, VA = Department of Veterans Affairs, $\mathrm{VO}_{2}=$ oxygen consumption.

*Address all correspondence to Richard F. Macko, MD; Department of Neurology, Baltimore Department of Veterans Affairs Medical Center, 10 North Greene Street, Baltimore, MD 21201; 410-605-5413; fax: 410-605-7913.

Email: rmacko@grecc.umaryland.edu

DOI: 10.1682/JRRD.2006.10.0130 
body weight-supporting paradigm (Miyai et al. [3]). We were interested in evaluating whether a straightforward, progressive aerobic training program that could be easily translated to a community setting would be beneficial and safe. A small study $(n=4)$ supported the feasibility and efficacy of aerobic training in patients with mild PD [5]; however, prior studies did not investigate aerobic training in individuals who have significant gait impairment or postural instability as a result of PD. Additionally, questions about the safety and feasibility of an aerobic training regimen have not been adequately addressed. This study investigated the hypothesis that progressive treadmill aerobic exercise (TM-AEX) for 3 months is safe and feasible for patients with PD who exhibit gait impairment. Improvements in disease severity, ambulatory function, and cardiovascular fitness were also evaluated.

\section{METHODS}

\section{Subjects}

Neurologists trained in evaluating movement disorders referred individuals meeting the standard accepted United Kingdom Parkinson's Disease Society Brain Bank criteria for a definite diagnosis of idiopathic PD [6]. These standard Brain Bank criteria for PD diagnosis include bradykinesia and at least one of the following: (1) muscular rigidity; (2) 4 to $6 \mathrm{~Hz}$ resting tremor; and (3) postural instability not caused by primary visual, vestibular, cerebellar, or proprioceptive dysfunction. We also required at least three of the following eight supportive features to definitively diagnose PD: (1) unilateral onset, (2) presence of rest tremor, (3) progressive disorder, (4) persistent asymmetry most affecting the side of onset, (5) excellent (70\%-100\%) response to levodopa, (6) severe levodopainduced chorea, (7) levodopa response for $\geq 5$ years, and (8) clinical course of $\geq 10$ years. The individuals selected for participation did not have any of the 15 standard Brain Bank diagnostic criteria that exclude a PD diagnosis (such as rapidly progressive dementia and gaze palsy).

Subjects were recruited from the Baltimore Department of Veterans Affairs (VA) Medical Center and the University of Maryland PD and Movement Disorders Center. The University of Maryland School of Medicine Institutional Review Board approved the protocol, and we obtained informed consent from all subjects. The main inclusion criteria were diagnosis of levodoparesponsive PD; Hoehn and Yahr (HY) stage $\geq 2$; and presence of gait impairment, including dragging of the affected leg, freezing, or festination. We performed all evaluations while the subjects were "on" within 3 hours of medication intake. We requested subjects to come in for evaluations in the on state and used an additional dose of medication to maintain the on state for assessment and training when necessary.

Subjects were screened for dementia and depression and evaluated with the Unified Parkinson's Disease Rating Scale (UPDRS) [7], HY scale [8], and Schwab and England Activities of Daily Living Scale. We excluded patients with Mini-Mental State Examination [9] scores of $<24$, untreated depression, or medical/orthopedic conditions that would interfere with exercise.

\section{Treadmill Exercise Testing}

Eligible individuals first underwent an initial zeroincline treadmill tolerance test to evaluate their ability to ambulate and then a screening treadmill stress test (graded treadmill exercise testing [GXT]). A protocol based on a modified Bruce protocol was used for the initial GXT. The GXT was started at the subject's selfselected comfortable walking speed. Then, treadmill speed or incline was increased according to a protocol that we validated for use with stroke patients [10-12]. All subjects wore a gait belt to improve stability, and a spotter stood behind subjects during both treadmill evaluations.

Subjects without signs of cardiac ischemia on the screening GXT were evaluated with a progressive GXT on a separate day at least 1 week later. During the progressive GXT, subjects exercised to peak volitional fatigue (peak effort). We used open circuit spirometry to measure oxygen consumption $\left(\mathrm{VO}_{2}\right)$ peak [11-12]. We performed two $\mathrm{VO}_{2}$ peak evaluations and used the highest heart rate (HR) of the three evaluations (including the GXT or either of the two $\mathrm{VO}_{2}$ peaks) to determine the subject's target peak HR. Estimated maximal treadmill walking workload capacity (expressed in estimated metabolic equivalents [METs]) was calculated from the treadmill incline and velocity during the progressive GXT. Estimated METs were calculated from the treadmill speed and incline based on American College of Sports Medicine guidelines.

\section{Gait and Balance Evaluations}

We used four tests to assess ambulatory function preand postintervention. The first test consisted of a $9.1 \mathrm{~m}$ walk. In this test, each subject walked at a self-selected pace. Mean velocities (meters per second) based on an average of three trials were calculated from three timed trials separated by a rest interval. In a second test, after a 
5 min rest interval, the first test was repeated at the subject's fastest comfortable pace. For the third test, after 20 min of rest, the subjects performed a timed 6 min walk and we measured the distance traveled around a $30.5 \mathrm{~m}$ track. In the final test, we determined routine daily ambulatory activity by using a microprocessor-linked step activity monitor (Cyma Corporation; Mountlake Terrace, Washington) that made a $48 \mathrm{~h}$ recording of the number of strides. The pager-sized stride counter was strapped to the subject's right leg above the lateral malleolus. We compared visually counted strides with step activity monitor- counted strides during a 1 min floor walk to validate accuracy for each subject [13].

\section{Treadmill Aerobic Exercise Training}

Subjects started the TM-AEX training at their comfortable walking speed for 10 to $20 \mathrm{~min}$, with training parameters altered according to their ability to tolerate the exercise. We monitored cardiac safety and initially ensured that subjects did not exceed 10 to $20 \mathrm{~min}$ at 40 to 50 percent of maximal HR reserve, as calculated by the Karvonen formula [12]:

$$
T=\left(\mathrm{HR}_{\max }-\mathrm{HR}_{\mathrm{rest}}\right) \times(\text { percent intensity })+\mathrm{HR}_{\mathrm{rest}} \quad \text {, }
$$

where $T=$ target $\mathrm{HR}, \mathrm{HR}_{\max }=$ maximal $\mathrm{HR}$, and $\mathrm{HR}_{\mathrm{rest}}$ = resting HR. We described use of the Karvonen formula to determine peak exercise capacity in our previous work with stroke patients [10-11]. In this case, $\mathrm{HR}_{\max }$ and $\mathrm{HR}_{\text {rest }}$ were taken from the subjects' best performance on either the GXT or one of the two $\mathrm{VO}_{2}$ peak evaluations before training. Subjects incapable of continuous training performed bouts of intermittent exercise separated by rest to achieve 10 to $20 \mathrm{~min}$ of training. Initial velocities were determined by functional gait parameters, with most subjects beginning walking at their comfortable overground walking pace. Every 2 weeks as tolerated, we increased duration by $5 \mathrm{~min}$ and intensity by $0.1 \mathrm{mph}$ and/or incline by 1 percent as tolerated. For safety reasons, subjects were not allowed to exceed 70 percent of their HR reserve as calculated by the Karvonen formula. Blood pressure and HR were taken before exercise, at the midpoint of exercise, and after exercise. Subjects were supported by either a Biodex ${ }^{\mathrm{TM}}$ harness (Biodex Medical Systems, Inc; Shirley, New York) or a TreadAbout (Innovative Health Solutions; League City, Texas). No body-weight support was provided. Subjects could use handrails for balance if necessary but were encouraged to attempt to train without using handrails. For all subjects, training was started at a walking pace. One subject (HY stage 2) was able to jog toward the end of training. Training was performed three times a week for 12 weeks.

\section{Postexercise Testing}

A disease-specific measure of disease severity (the UPDRS) was repeated after 3 months of TM-AEX. Cardiovascular and gait measures, including ambulatory measures, were also repeated after the 3-month training regimen. A fellowship-trained movement disorders physician performed the neurological evaluations. Because this was a pilot safety and feasibility study, the pre- and postevaluations were unblinded.

\section{Data Analysis}

Falls or near falls and systolic blood pressure (SBP) changes $>20 \mathrm{mmHg}$ during training sessions were documented. A fall was defined as postural instability, cessation of treadmill operation by an assistant, and inability to recover balance without assistance. A near fall was defined as postural instability with or without cessation of treadmill operation but no need for assistance to recover balance. Data are expressed as mean \pm standard deviation. Significance was set at $p<0.05$ (two-tailed).

We used paired Student's $t$-tests to compare $\mathrm{VO}_{2}$ peak, 6 min walk, $9.1 \mathrm{~m}$ walks, and step activity evaluations before and after training. Wilcoxon signed rank tests evaluated differences in UPDRS before and after training. All analyses were performed with SPSS version 13.0 (SPSS, Inc; Chicago, Illinois).

\section{RESULTS}

\section{Demographics and Initial Screening Treadmill Exercise Testing}

Eight individuals underwent the screening GXT. Three subjects were deemed ineligible to enroll in the training protocol: two were referred to a cardiologist for asymptomatic ST segment depression and one for a symptomatic $30 \mathrm{mmHg}$ drop in SBP during the screening GXT. In both cases of asymptomatic ST segment depression, after a cardiac workup, the subjects were deemed eligible to participate but the study enrollment period had ended. No cause for the significant exercise intolerance was discovered for the third ineligible subject; however, the subject later developed significant executive dysfunction and autonomic instability, raising the possibility that 
he had levodopa-responsive parkinsonism rather than PD. No final diagnosis, however, is available because the patient was lost to follow-up. Five subjects enrolled in the study. Demographics for all screened subjects are presented in Table 1. Screened subjects reached peak effort at a velocity of $0.7 \pm 0.2 \mathrm{~m} / \mathrm{s}, 11 \pm 4$ percent grade, and $9.2 \pm 2.6 \mathrm{~min}$, achieving a mean $72 \pm 10$ percent of their age-predicted maximum HR.

\section{Treadmill Aerobic Exercise Training Response}

Target treadmill velocity was determined by the subject's cardiovascular parameters $\left(\mathrm{VO}_{2}\right.$ peak, peak ambulatory work capacity as defined by initial estimated METs, and self-selected walking speed). In two subjects, difficulties with gait coordination on the treadmill necessitated slow initial velocities. The cohort made steady advances in treadmill speed and peak ambulatory work capacity over the 3-month period (Table 2). Table 3 shows individual subject gains in treadmill speed, grade, and duration during the 3 months of TM-AEX training.

\section{Safety}

Nine episodes of SBP drops $>20 \mathrm{mmHg}$ occurred in 136 exercise sessions. Two subjects had one asymptomatic episode each, while one subject had seven episodes (six asymptomatic and one symptomatic). In this subject (subject 1, HY stage 2), the symptomatic episode prompted a 3-week study interruption for cardiac evaluation. This subject experienced normal SBP elevations at exercise intensities that at other times had resulted in SBP drops. This subject had marked motor fluctuations, the highest baseline $\mathrm{VO}_{2}$ peak, and the lowest baseline "on" UPDRS III (motor) score. No correlation between motor fluctuations and SBP changes was observed. Three weeks were added to the end of training to make up for the break in training. Two subjects (Subjects 5 and 6, HY stages 3 and 4, respectively) experienced four falls and

Table 1.

Demographic characteristics of subjects with Parkinson disease (PD) screened for participation in treadmill aerobic exercise study.

\begin{tabular}{|c|c|c|c|c|c|c|c|c|c|c|}
\hline \multirow[b]{2}{*}{ Category } & \multicolumn{8}{|c|}{ Individual Subjects } & \multicolumn{2}{|c|}{ All Subjects (mean \pm SD) } \\
\hline & $\mathbf{1}^{*}$ & 2 & 3 & $4^{*}$ & $5^{*}$ & $6^{*}$ & $7^{*}$ & 8 & $\begin{array}{c}\text { Screened } \\
(n=8)\end{array}$ & $\begin{array}{c}\text { Enrolled } \\
(n=5)\end{array}$ \\
\hline Age (yr) & 69 & 70 & 62 & 71 & 78 & 61 & 57 & 70 & $67 \pm 7$ & $67 \pm 8$ \\
\hline Sex & M & $\mathrm{M}$ & M & $\mathrm{M}$ & M & $\mathrm{F}$ & $\mathrm{M}$ & M & $7 / 1^{\dagger}$ & $4 / 1^{\dagger}$ \\
\hline \multicolumn{11}{|l|}{ UPDRS } \\
\hline Total & 44 & 22 & 24 & 52 & 64 & 53 & 54 & 113 & $53 \pm 28$ & $53 \pm 7$ \\
\hline Cognitive (I) & 2 & 2 & 0 & 4 & 6 & 3 & 4 & 7 & $4 \pm 2$ & $4 \pm 1$ \\
\hline ADL (II) & 17 & 3 & 5 & 17 & 19 & 15 & 18 & 30 & $19 \pm 18$ & $17 \pm 1$ \\
\hline \multicolumn{11}{|l|}{ Cardiovascular Parameters } \\
\hline Treadmill Rate (m/s) & 0.6 & 0.7 & 1.1 & 0.8 & 0.4 & 0.8 & 0.7 & 0.6 & $0.7 \pm 0.2$ & $0.7 \pm 0.2$ \\
\hline Treadmill Elevation (\%) & 16 & 10 & 12 & 8 & 12 & 14 & 14 & 2 & $11 \pm 4$ & $13 \pm 3$ \\
\hline Age-Predicted Max HR & 151 & 150 & 158 & 149 & 142 & 159 & 163 & 150 & $153 \pm 7$ & $153 \pm 8$ \\
\hline Target HR (85\% max) & 128 & 128 & 134 & 127 & 121 & 135 & 139 & 128 & $130 \pm 6$ & $130 \pm 7$ \\
\hline Max HR During Testing & 128 & 84 & 120 & 104 & 85 & 134 & 124 & 105 & $110 \pm 19$ & $115 \pm 20$ \\
\hline
\end{tabular}


Table 2.

Training parameters for five subjects with advanced Parkinson disease who participated in 3-month treadmill aerobic exercise program. Data shown as mean \pm standard deviation.

\begin{tabular}{|c|c|c|c|c|c|c|c|}
\hline Exercise Parameter & Start & Week 2 & Week 4 & Week 6 & Week 8 & Week 10 & Week 12 \\
\hline $\begin{array}{l}\text { Treadmill Velocity (\% of } \\
\text { comfortable walk speed) }\end{array}$ & $72 \pm 13$ & $86 \pm 17$ & $98 \pm 8$ & $100 \pm 16$ & $104 \pm 16$ & $110 \pm 17$ & $114 \pm 19$ \\
\hline $\begin{array}{l}\text { Workload (\% of pretraining } \\
\text { estimated maximal METs) }\end{array}$ & $41 \pm 5$ & $50 \pm 9$ & $60 \pm 13$ & $64 \pm 14$ & $64 \pm 13$ & $74 \pm 17$ & $79 \pm 22$ \\
\hline
\end{tabular}

MET = metabolic equivalent.

Table 3.

Overall change in treadmill training parameters during 3-month treadmill aerobic exercise program. Data shown as percent increase (initial $\rightarrow$ final).

\begin{tabular}{lcccccc}
\hline \multicolumn{1}{c}{ Category } & Subject 1 & Subject 4 & Subject 5 & Subject 6 & Subject 7 & Average \\
\hline Velocity (m/s) & $59(2.2 \rightarrow 3.5)$ & $28(1.8 \rightarrow 2.3)$ & $109(1.1 \rightarrow 2.3)$ & $47(1.7 \rightarrow 2.5)$ & $65(2.0 \rightarrow 3.3)$ & $56\left(1.8 \rightarrow 2.8^{*}\right)$ \\
Grade (\%) & NA $(2 \rightarrow 4)$ & NA $(0 \rightarrow 2)$ & NA $(0 \rightarrow 3)$ & NA $(0 \rightarrow 5)$ & NA $(0 \rightarrow 6)$ & NA $\left(0.4 \rightarrow 4.0^{\dagger}\right)$ \\
Duration (min) & $50(20 \rightarrow 30)$ & $25(20 \rightarrow 25)$ & $75(20 \rightarrow 35)$ & $50(20 \rightarrow 30)$ & $50(20 \rightarrow 30)$ & $52\left(20 \rightarrow 31^{*}\right)$ \\
Distance $(\mathrm{km})$ & $163(1.1 \rightarrow 2.9)$ & $60(1.0 \rightarrow 1.6)$ & $250(0.6 \rightarrow 2.1)$ & $60(1.0 \rightarrow 1.6)$ & $145(1.1 \rightarrow 2.7)$ & $120\left(1.0 \rightarrow 2.2^{*}\right)$ \\
\hline
\end{tabular}

${ }^{*} p<0.005$.

${ }^{\dagger} p<0.01$

NA = not applicable.

seven near falls, respectively, during training (11 events in 65 exercise sessions). Subjects fell into the gait harnesses, preventing injury in all cases.

\section{Feasibility}

All enrolled subjects completed the training program. Subjects attended $>90$ percent of scheduled exercise sessions over the 3-month study, with the exception of the subject who had a 3-week break in training for cardiac evaluation. Two subjects took 1-week breaks for personal scheduling conflicts or minor medical reasons. Subjects' training progression is cataloged in Table 2.

\section{PD Impairment and Performance Measures}

Table 4 shows individual subject improvement before and after the intervention, as well as overall changes and statistical analysis on our small sample. UPDRS improved following 3 months of TM-AEX $(p<0.05)$. In this small sample, the UPDRS III (motor) score was correlated with the 6 min walk distances $(r=-0.917, p<0.05)$, fast timed $9.1 \mathrm{~m}$ walks $(r=0.97, p<0.01)$, and initial $\mathrm{VO}_{2}$ peaks $(r=-0.90, p<0.05)$. The $9.1 \mathrm{~m}$ walk speed did not significantly change. We found a trend toward increased 6 min walk distances and steps taken in $24 \mathrm{~h}$.

\section{Cardiovascular Fitness Measures}

For analysis, we compared the highest preexercise $\mathrm{VO}_{2}$ peak evaluation with the poststudy $\mathrm{VO}_{2}$ peak evaluation. $\mathrm{VO}_{2}$ peak did not change. Peak ambulatory workload capacity (measured as estimated METs achieved at $\mathrm{VO}_{2}$ peak) [12] increased 26 percent with TM-AEX ( $p<$ 0.02) (Table 4).

\section{DISCUSSION}

This study shows that a progressive TM-AEX training program is feasible in selected individuals with PD and gait impairment. We encountered two safety issues: hypotension and falls. Exercise-triggered declines in SBP occurred in 9 (7\%) of the 136 exercise sessions. Hypotensive episodes were not attributable to any known cardiac abnormalities and may be secondary to the autonomic dysfunction found to some degree in most patients with PD [14]. Previous research has reported that SBP often declines as PD progresses [15], which is believed to be associated with decreased noradrenaline when the individual is upright [16]. The response of noradrenaline to exercise in PD has not been studied, however. Levodopa and dopamine agonists can also reduce SBP [17], which 
JRRD, Volume 45, Number 1, 2008

Table 4.

Effects of treadmill aerobic exercise on pre- and posttraining impairments, disability, fitness, ambulatory function, and general health status of five subjects with Parkinson disease.

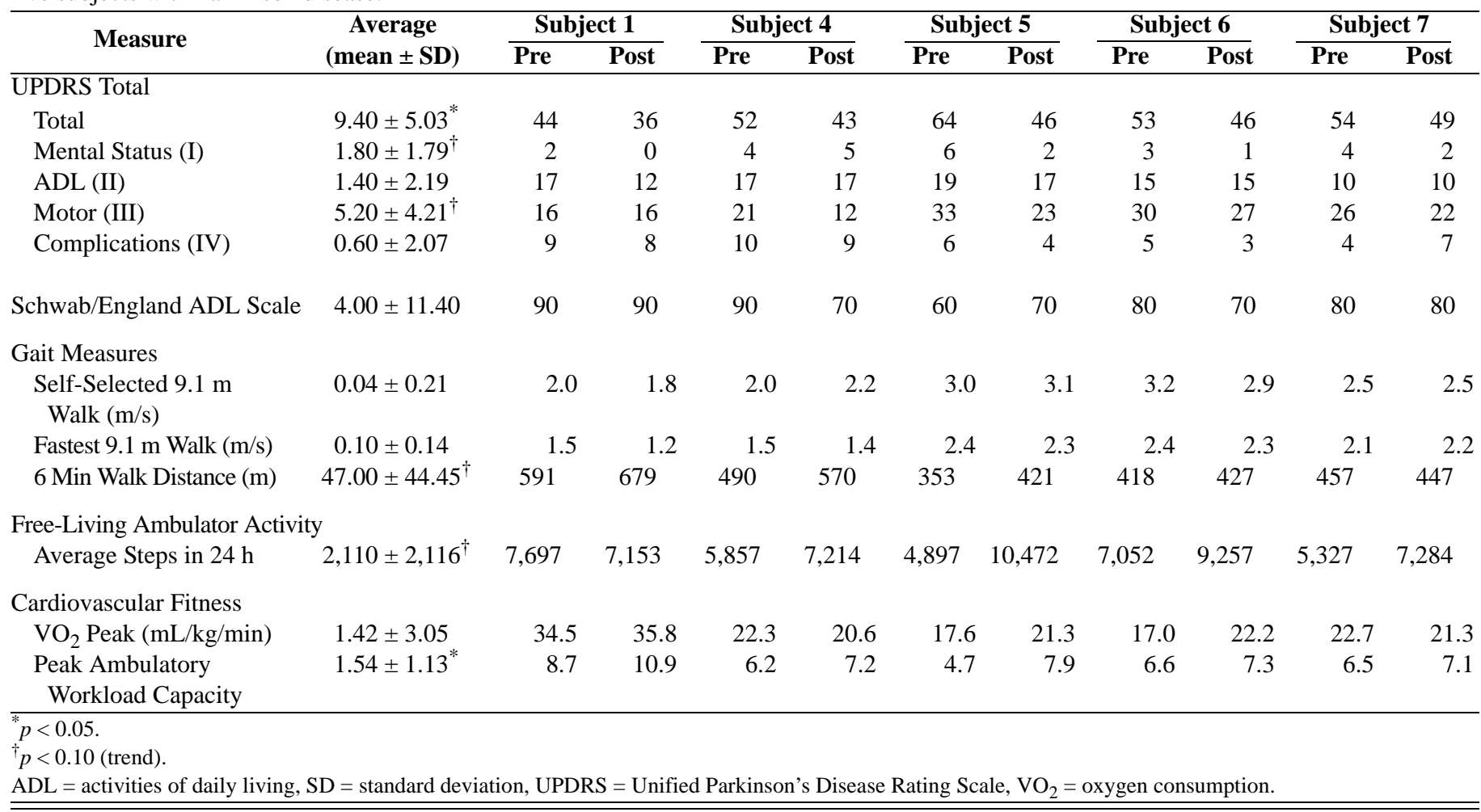

may complicate this population's cardiopulmonary response to exercise. Notably, of eight patients screened, three patients were unable to participate in the study because of findings on the initial exercise stress test. In two cases, unsuspected cardiac disease was discovered that required further evaluation. Both of these patients ultimately were cleared for participation in the study but enrollment had ended. In the third case, a significant symptomatic drop in SBP during the initial stress test suggested significant issues with exercise tolerance and mandated exclusion from the study. This excluded patient was levodopa responsive and diagnosed with probable idiopathic PD by a movement disorders specialist; however, a subsequent relatively rapid rate of progression of executive dysfunction and autonomic instability raises the possibility of parkinsonism, including a more diffuse Lewy body process or multiple systems atrophy. Although we screened a small number of patients, this relatively high rate of exclusion based on initial cardiac testing is interesting and merits further study. For example, as exercise is studied further, individuals with other causes of levodoparesponsive parkinsonism might be a part of the subject pool as a result of diagnostic uncertainty. Carefully monitored larger studies, therefore, need to define the cardiac risk that exercise may pose for patients with a PD diagnosis, characterize the impact of autonomic dysfunction on exercise tolerance, and optimize exercise regimens for patients with PD.

Disease severity, as measured by the UPDRS, improved after training. TM-AEX may improve neurological function in PD as a result of improved external cueing that facilitates gait patterning; this finding has been reported for spinal cord injury [18-19], stroke [20-21], and PD [2]. TM-AEX may also stimulate task-repetitive locomotor relearning [21]. Other potential mechanisms of improvement include musculoskeletal conditioning and aerobic fitness. In animal models of PD, TM-AEX has resulted in neuroprotective effects, preventing dopaminergic neuronal degeneration in 6-hydroxydopamine rats and 1-methyl-4phenyl-1,2,5,6-tetrahydropyridine mice [22]. Exerciserelated neuroprotection may be associated with induction of glial cell-derived neurotrophic factor [14], a growth factor known to be a potent neuroprotectant for dopaminergic neurons. In a prospective study of 48,574 men and 
77,254 women, Chen et al. found that the men's greater baseline physical activity was associated with a lower risk of developing PD [23]. An important, unanswered question is whether exercise interventions can potentially modify growth factors and neurochemistry in individuals with PD and thus improve motor control or alter the natural history of the functional decline.

Few studies have investigated whether task-repetitive exercise increases daily mobility and function in PD. TM-AEX increased peak ambulatory workload capacity 26 percent, similar to results reported with exercise training in subjects with stroke [10-11]. Notably, despite the subjects' improved peak ambulatory workload capacity, we observed no changes in mean $\mathrm{VO}_{2}$ peak, a wellestablished index of cardiovascular fitness [12]. This finding is not unexpected, because our experience with patients with stroke is that functional motor gains (and related peak ambulatory workload capacity) improve before cardiovascular improvement is observed. While $\mathrm{VO}_{2}$ peak is a good measure of cardiovascular fitness, it does not measure musculoskeletal components of fitness that are likely to be defective in PD. Patients may need to improve musculoskeletal fitness (as measured by estimated METs) before they can improve $\mathrm{VO}_{2}$ peak. This finding, seen in our experience with stroke patients, suggests that longer training with neurological populations may be required for functional motor gains to improve enough to allow cardiovascular improvement [10-11].

Functional ambulatory capacity improved in our small cohort. Microprocessor-linked step-activity monitoring showed a 34-percent increase in daily step activity, suggesting that TM-AEX may translate into improved mobility. Our findings suggest a use for portable monitoring of free-living ambulatory activity in PD and provide evidence that daily ambulation may be modifiable with exercise.

\section{CONCLUSIONS}

This pilot study demonstrated that TM-AEX is feasible in selected PD subjects with gait impairment. Hypotensive episodes and falls were identified as safety concerns, and we noted that three of eight patients could not participate because of issues identified during the initial cardiac stress testing. Among the subjects who participated, the intervention improved their walking speed, home ambulation, peak ambulatory workload capacity, and UPDRS scores. Generalizability of these results is limited since the study was a single-arm, unblinded study with a small sample size. Randomized studies are needed for a better understanding of the potential for task-oriented exercise training to improve locomotor function and metabolic fitness.

\section{ACKNOWLEDGMENTS}

Dr. Skidmore is now with the Department of Neurology, University of Florida School of Medicine, Gainesville, Florida.

This material was based on work supported by the VA Research and Development Center of Excellence in Task-Oriented Exercise and Robotics in Neurological Disease; the VA Stroke Research Enhancement Award Program; the VA Geriatrics Research, Education, and Clinical Center; the Rosalyn Newman Foundation; the University of Maryland Claude D. Pepper Older Americans Independence Center (grant P60AG12583); and a Medtronics Movement Disorders Fellowship.

The authors have declared that no competing interests exist.

\section{REFERENCES}

1. Sofuwa O, Nieuwboer A, Desloovere K, Willems AM, Chavret F, Jonkers I. Quantitative gait analysis in Parkinson's disease: Comparison with a healthy control group. Arch Phys Med Rehabil. 2005;86(5):1007-13.

[PMID: 15895349]

2. Frenkel-Toledo S, Giladi N, Peretz C, Herman T, Gruendlinger L, Hausdorff JM. Treadmill walking as an external pacemaker to improve gait rhythm and stability in Parkinson's disease. Mov Disord. 2005;20(9):1109-14. [PMID: 15929090]

3. Miyai I, Fujimoto Y, Yamamoto H, Ueda Y, Saito T, Nozaki S, Kang J. Long-term effect of body weightsupported treadmill training in Parkinson's disease: A randomized controlled trial. Arch Phys Med Rehabil. 2002; 83(10):1370-73. [PMID: 12370870]

4. Protas EJ, Mitchell K, Williams A, Qureshy H, Caroline K, Lai EC. Gait and step training to reduce falls in Parkinson's disease. NeuroRehabilitation. 2005;20(3):183-90. [PMID: 16340099]

5. Bergen JL, Toole T, Elliott RG 3rd, Wallace B, Robinson K, Maitland CG. Aerobic exercise intervention improves aerobic capacity and movement initiation in Parkinson's 
disease patients. NeuroRehabilitation. 2002;17(2):161-68. [PMID: 12082243]

6. Gibb WR, Lees AJ. The relevance of the Lewy body to the pathogenesis of idiopathic Parkinson's disease. J Neurol Neurosurg Psychiatry. 1988;51(6):745-52. [PMID: 2841426]

7. Martínez-Martín P, Gil-Nagel A, Gracia LM, Gómez JB, Martínez-Sarriés J, Bermejo F. Unified Parkinson’s Disease Rating Scale characteristics and structure. The Cooperative Multicentric Group. Mov Disord. 1994;9(1):76-83. [PMID: 8139608]

8. Hoehn MM, Yahr MD. Parkinsonism: Onset, progression and mortality. Neurology. 1967;17(5):427-42. [PMID: 6067254]

9. Folstein MF, Folstein SE, McHugh PR. "Mini-mental state." A practical method for grading the cognitive state of patients for the clinician. J Psychiatr Res. 1975;12(3):189-98. [PMID: 1202204]

10. Macko RF, Ivey FM, Forrester LW, Hanley D, Sorkin JD, Katzel LI, Silver KH, Goldberg AP. Treadmill exercise rehabilitation improves ambulatory function and cardiovascular fitness in patients with chronic stroke: A randomized, controlled trial. Stroke. 2005;36(10):2206-11. [PMID: 16151035]

11. Macko RF, Smith GV, Dobrovolny CL, Sorkin JD, Goldberg AP, Silver KH. Treadmill training improves fitness reserve in chronic stroke patients. Arch Phys Med Rehabil. 2001;82(7):879-84. [PMID: 11441372]

12. Whaley MH, Brubaker PH, Otto RM, Armstrong LE. ACSM's guidelines for exercise testing and prescription. 7th ed. Philadelphia (PA): Lippincott Williams \& Wilkins; 2006. p. 286-99.

13. Macko RF, Haeuber E, Shaughnessy M, Coleman KL, Boone DA, Smith GV, Silver KH. Microprocessor-based ambulatory activity monitoring in stroke patients. Med Sci Sports Exerc. 2002;34(3):394-99. [PMID: 11880800]

14. Smith AD, Zigmond MJ. Can the brain be protected through exercise? Lessons from an animal model of parkinsonism. Exp Neurol. 2003;184(1):31-39. [PMID: 14637076]
15. Schrag A, Ben-Shlomo Y, Quinn N. How common are complications of Parkinson's disease? J Neurol. 2002;249(4): 419-23. [PMID: 11967646]

16. Marttila RJ, Rinne UK. Level of blood pressure in patients with Parkinson's disease. A case-control study. Eur Neurol. 1977;16(1-6):73-78. [PMID: 354939]

17. Turkka JT. Correlation of the severity of autonomic dysfunction to cardiovascular reflexes and to plasma noradrenaline levels in Parkinson's disease. Eur Neurol. 1987;26(4): 203-10. [PMID: 3595658]

18. Barbeau H, Rossignol S. Recovery of locomotion after chronic spinalization in the adult cat. Brain Res. 1987; 412(1):84-95. [PMID: 3607464]

19. Pépin A, Norman KE, Barbeau H. Treadmill walking in incomplete spinal-cord-injured subjects: 1 . Adaptation to changes in speed. Spinal Cord. 2003;41(5):257-70. [PMID: 12714988]

20. Hesse S, Konrad M, Uhlenbrock D. Treadmill walking with partial body weight support versus floor walking in hemiparetic subjects. Arch Phs Med Rehabil. 1999;80(4): 421-27. [PMID: 10206604]

21. Forrester LW, Handley DF, Macko RF. Effects of treadmill exercise on transcranial magnetic stimulation-induced excitability to quadriceps after stroke. Arch Phys Med Rehabil. 2006;87(2):229-34. [PMID: 16442977]

22. Tillerson JL, Caudle WM, Reverón ME, Miller GW. Exercise induces behavioral recovery and attenuates neurochemical deficits in rodent models of Parkinson's disease. Neuroscience. 2003;119(3):899-911. [PMID: 12809709]

23. Chen H, Zhang SM, Schwarzschild MA, Hernán MA, Ascherio A. Physical activity and the risk of Parkinson disease. Neurology. 2005;64(4):664-69. [PMID: 15728289]

Submitted for publication October 24, 2006. Accepted in revised form July 23, 2007. 